\section{International Scientific Journal Theoretical \& Applied Science}

p-ISSN: 2308-4944 (print) $\quad$ e-ISSN: 2409-0085 (online)

Year: 2015 Issue: 04 Volume: 24

Published: $30.04 .2015 \quad$ http://T-Science.org
Sergey Aleksandrovich Vorobyev

Candidate of Technical Sciences, associate professor,

Don State Technical University, Russia v.serge.79@mail.ru

Sergey Sergeevich Vorobyev

Candidate of Technical Sciences, associate professor, Don State Technical University, Russia

Andrey Stanislavovich Reshenkin

Candidate of Technical Sciences, head of department,

Don State Technical University, Russia

Elena Victorovna Malaja

Candidate of Technical Sciences, associate professor, Don State Technical University, Russia

Alexander Grigoryevich Tihomirov

Candidate of Technical Sciences, associate professor,

Don State Technical University, Russia

\title{
ELEMENTS OF THE APPLIED THEORY OF OPTIMAL VIBRATION DAMPING
}

Abstract: The paper presents the research in the development of the effective systems of the vehicle suspension protection undergoing the dynamic effects of the support surface irregularities. These results may be useful for the professionals of other areas associated with both applications of the theory of optimal vibration damping and the applications of the theory of optimum control of other processes.

Key words: applied theory, vehicle suspension, damping.

Language: Russian

Citation: Vorobyev SA, Vorobyev SS, Reshenkin AS, Malaja EV, Tihomirov AG (2015) ELEMENTS OF THE APPLIED THEORY OF OPTIMAL VIBRATION DAMPING. ISJ Theoretical \& Applied Science 04 (24): 149-154.
Soi: $\underline{\text { http://s-o-i.org/1.1/TAS*04(24)26 }}$
Doi: crossef http://dx.doi.org/10.15863/TAS.2015.04.24.26

\section{ЭЛЕМЕНТЫ ПРИКЛАДНОЙ ТЕОРИИ ОПТИМАЛЬНОГО ДЕМПФИРОВАНИЯ КОЛЕБАНИЙ}

Аннотация: В статье приведены исследования в рамках разработки эффективных систем защиты подвески автомобилей, подвергающихся динамическому воздействию неровностей опорной поверхности. Эти результаты также могут быть полезнь спечиалистам других направлений, связанных как с приложениями теории оптимального демпфирования колебаний, так и с приложениями теории оптимального управления другими процессами.

Ключевые слова: прикладная теория, подвеска автомобиля, демпфирование.

При решении научных задач в качестве физических моделей объектов исследования принимаются системы с сосредоточенными параметрами, представляющие собой совокупность абсолютно твердых тел, соединенных друг с другом и основанием упругим подвесом [1-2]. Их математическими моделями являются системы обыкновенных дифференциальных уравнений, полученных в обобщенных координатах на основе интегрального принципа ГамильтонаОстроградского или дифференциального принципа Лагранжа второго рода. Вывод условий оптимальности осуществляется методом принципа максимума Понтрягина.

Под оптимальными в работе понимаются такие алгоритмы управления жесткостью, при которых энергия колебательного движения снижается до заданной величины за минимальное время. Задача формулируется так [3]:

Для колебательного процесса, описываемого системой уравнений (1) при начальных условиях (2) определить алгоритм управления матрицей квазиупругих коэффициентов, чтобы изменение кинетической энергии колебательного движения от начального значения $\mathrm{T}_{(0)}$ до заданного конечного $\mathrm{T}_{(\mathrm{k})}$ происходило за минимальное время (3). При этом на коэффициенты жесткости могут накладываться ограничения типа неравенства (4).

$$
\begin{gathered}
A \ddot{z}+C z=q(t, C) \\
t=0, z=z_{(0)}, \dot{z}=\dot{z}_{(0)}, T_{0}=\frac{1}{2} \dot{z}_{(0)}^{T} A \dot{z}_{(0)}
\end{gathered}
$$




$$
\begin{gathered}
t=t_{(k)}, \quad T_{(k)}=\frac{1}{2}\left(\dot{z}_{(k)}^{T} A \dot{z}_{(k)}\right) ; \\
J=\int_{0}^{t_{(k)}} d t \rightarrow \min \\
c_{i k} \in\left\lfloor\left(c_{i k}\right)_{\min ,}\left(c_{i k}\right)_{\max }\right\rfloor .
\end{gathered}
$$

Здесь $\mathbf{Z}, \dot{z}$ - векторы обобщенных координат и обобщенных скоростей, размерностью $\mathrm{k} \times 1$ каждый; A - матрица коэффициентов инерции размерностью $\mathrm{n} \times \mathrm{n}$; C- матрица квазиупругих коэффициентов $\mathrm{n} \times \mathrm{n} ; \mathrm{q}(\mathrm{t}, \mathrm{C}) \quad-\quad$ вектор обобщенной силы размерностью $\mathrm{n} \times 1$; $\mathrm{T}-$ кинетическая энергия колебательного движения системы; (o),(k)- индексы, означающие начальное и конечное состояния.

Такая формулировка задачи обусловлена тем, что кинетическая энергия является квадратичной формой обобщенных скоростей и характеризует интенсивность колебательного, динамического процесса. Скорость ее убыли будет характеризовать эффективность гашения колебаний и, естественно, за минимальное время конструкция будет испытывать меньшее число толчков и знакопеременных нагружений [4-6].

Для определения условий оптимальности закона изменения жесткости использовалось понятие игольчатой вариации (рис.1) и стандартное расширение фазового вектора $\mathrm{z}(\mathrm{t})$ путем переобозначения функционала $\mathrm{J}$ и времени $t$ :

$$
\begin{gathered}
z=\left(z^{0}, z^{1}, \ldots, z^{n}, z^{n+1}\right) \\
z^{0} \equiv J, \quad \dot{z}^{0}=1 ; \quad z^{n+1} \equiv t, \quad \dot{z}^{n+1}=1
\end{gathered}
$$

Путем введения $\mathrm{n}+2$ - мерного вектора сопряженных функций $\psi=\left(\psi_{0}, \psi_{1}, \ldots, \psi_{\mathrm{n}}, \psi_{\mathrm{n}+1}\right)^{\mathrm{T}}$ для фазовых координат и множителя Лагранжа $\lambda$ для краевого условия $t=t_{(\kappa)}$ вместо исходного функционала строился расширенный функционал по правилу

$$
\begin{aligned}
& J^{*}=\int_{0}^{t_{k}}\left\{\begin{array}{l}
\left(\dot{z}^{0}-1\right) \psi_{0}+ \\
\left.\sum_{i, j=1}^{n} \psi_{i}\left[A_{i j} \ddot{z}^{j}+c_{i j} z^{j}-q_{i}\left(Z^{n+1}, C\right)\right]\right\} d t \\
+\left(\dot{z}^{n+1}-1\right) \psi_{n+1}
\end{array}\right] \\
& \quad+\lambda\left(T_{(k)}-T_{(0)}\right)= \\
& =\int_{0}^{\mathrm{t}_{\mathrm{k}} \mathrm{F}(\mathrm{z}, \psi, \mathrm{C}) \mathrm{dt}+\lambda\left(\mathrm{T}_{(\mathrm{k})}-\mathrm{T}_{(0)}\right)}
\end{aligned}
$$

где $\mathrm{F}(\mathrm{z}, \psi, \mathrm{C})$ - подинтегральная функция.

Представление игольчатой вариации как логической разности между варьированным $C_{\varepsilon}$ и неварьированным $\widetilde{C}$ значением коэффициентов упругости (управления) (рис.1)

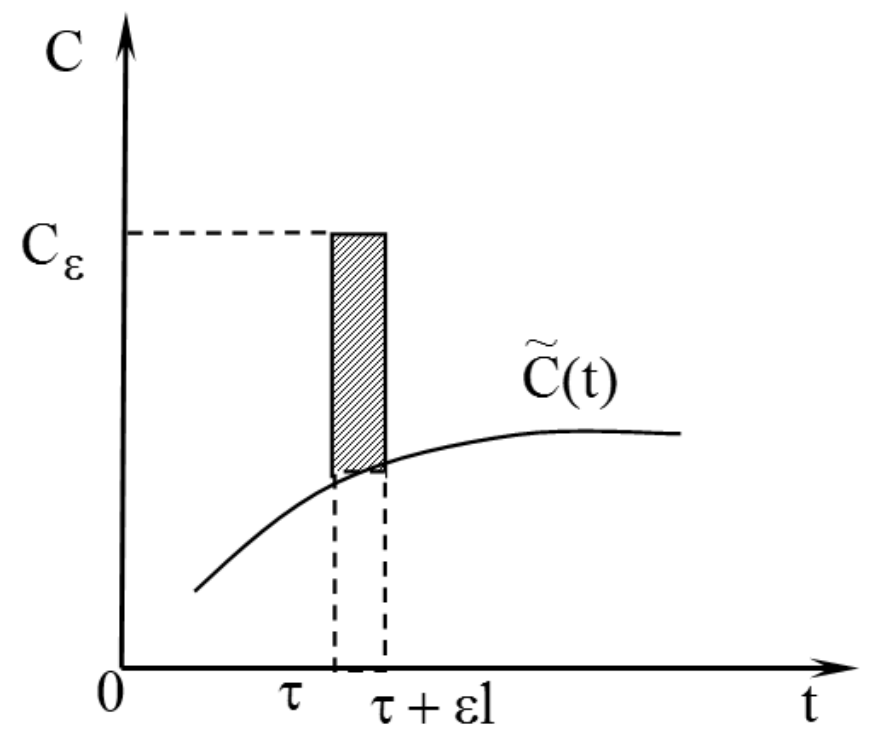

Рисунок 1 - Игольчатая вариация управления. 


$$
\begin{gathered}
\delta C=C_{\varepsilon} \backslash \tilde{C} ; \\
\mathrm{C}_{\varepsilon}(\mathrm{t})=\left\{\begin{array}{ll}
\mathrm{C}(\mathrm{t}), & \mathrm{t} \in[\tau, \tau+\varepsilon \mathrm{l}] ; \\
\widetilde{\mathbf{C}}(\mathrm{t}), & \mathrm{t} \notin[\tau, \tau+\varepsilon l] ;
\end{array} \varepsilon\langle 1,\right.
\end{gathered}
$$

позволяет определить главную часть приращения расширенного функционала.

Для этого расширенный функционал можно представить в виде суммы трех интегралов на временных интервалах

$$
\begin{aligned}
& t \in[O, \tau], t \in[\tau, \tau+\varepsilon l] \\
& t \in\left[\tau+\varepsilon l, t_{(k)}\right]
\end{aligned}
$$

$$
\begin{aligned}
& J_{\varepsilon}^{*}=\int_{0}^{\tau} F_{\varepsilon} d t+\int_{\tau}^{\tau+\varepsilon l} F_{\varepsilon} d t+ \\
& \int_{\tau+\varepsilon l}^{t_{(k)}} F_{\varepsilon} d t+\lambda\left(T_{\varepsilon(k)}-T_{0}\right) \\
& \widetilde{J}^{*}=\int_{0}^{\tau} \tilde{F} d t+\int_{\tau}^{\tau+\varepsilon l} \tilde{F} d t+ \\
& \int_{\tau+\varepsilon l}^{t_{(k)}} \tilde{F} d t+\lambda\left(\widetilde{T}_{(k)}-T_{(0)}\right)
\end{aligned}
$$

Тогда главная часть приращения функционала равна разности между варьированным и неварьированным значениями расширенного функционала

$$
\begin{aligned}
& \Delta J=J_{\varepsilon}^{*}-\tilde{J}^{*}=\varepsilon l\left[F\left(z, C_{\varepsilon}, \psi\right)-F(z, \tilde{C}, \psi)\right]+ \\
& +\int_{\tau+\varepsilon l}^{t_{(k)}}\left[F\left(z_{\varepsilon}, \tilde{C}, \psi\right)-F(\tilde{z}, \tilde{C}, \psi)\right] d t+\lambda\left(T_{\varepsilon(k)}-\tilde{T}_{(k)}\right) .
\end{aligned}
$$

Учитывая, что на первом интервале $\mathrm{t} \in[0, \tau]$ управления неварьированы и поэтому интегралы одинаковы; на втором интервале $\mathrm{t} \in[\tau, \tau+\varepsilon \mathrm{l}]$ приращение функционала получается только за счет игольчатой вариации управления (жесткости); на третьем интервале $\mathrm{t} \in\left\lfloor\tau+\varepsilon l, \mathrm{t}_{(\mathrm{k})}\right\rfloor$ управления не варьированы, а приращения функционала получается за счет вариаций обобщенных координат. Тогда после соответствующих преобразований получим необходимые и достаточные условия оптимальности в форме теоремы 1 принципа максимума [7-8].

Tеорема 1. Для того, чтобы управления $\mathrm{C}(t)$ и соответствующие им обобщенные координаты $\mathrm{z}(t)$ доставляли минимум функционалу быстродействия при уменьшении кинетической энергии до заданного конечного значения, необходимо и достаточно существование ненулевой непрерывной вектор-функций $\psi(t)$, удовлетворяющей сопряженной системе уравнений

$$
\begin{array}{r}
\dot{\psi}_{0}=0 ; \dot{\psi}_{\mathrm{n}+1}=-\sum_{\mathrm{i}=1}^{\mathrm{n}} \psi_{\mathrm{i}} \dot{\mathrm{q}}_{\mathrm{i}} ; \\
\sum_{i=1}^{n}\left(\ddot{\psi}_{i} A_{i j}+\psi_{i} c_{i j}\right)=0, j=\overline{1, n},
\end{array}
$$

и условиям трансверсальности в конечный момент времени

$$
\begin{gathered}
t=t_{(k)}, \psi_{0}=1 ; \psi_{i(k)}=-\lambda \dot{z}_{(k)}^{i}, \\
\psi_{n+1}=0, i=\overline{1, n}
\end{gathered}
$$

а функция Гамильтона

$$
H(z, \psi, \stackrel{\circ}{C})=\sum_{i, j=1}^{n} \psi_{i}\left[c_{i j} \dot{z}^{j}-q_{i}(C)\right]=\max _{C \in \bar{C}} H(\psi, t, C)
$$

при любом $t \in\left[\mathrm{O}, t_{(k)}\right]$ достигала своего максимального значения по всем $\mathrm{C} \in \overline{\mathrm{C}}$. При этом множитель Лагранжа постоянен, а его знак определяется целью задачи.
Таким образом, задача об определении оптимального закона (алгоритма) демпфирования колебаний сводится к решению двухточечной краевой задачи (уравнения 1-4, 11-13), в которой начальные данные для обобщенных координат заданы в начальный момент времени, а для

ISPC The Combination of Technology $\&$ 
сопряженных переменных в конечный момент времени.

Это является существенной трудностью как для получения решений оптимизационной задачи, так и технической реализации полученных алгоритмов в реальном масштабе времени.

Преодолеть эту трудность возможно с помощью метода мгновенно оптимального управления. Идея метода принадлежит В. Комкову. Заключается он в том, что определение оптимального управления осуществляется не в терминах конечного состояния системы, а по текущему ее состоянию. Базируется он на двух положениях.

Первое положение заключается в том, что задача об оптимальном по быстродействию заданном изменении кинетической энергии адекватна задаче о максимальном изменении кинетической энергии на заданном промежутке времени.

Второе положение позволяет обосновать возможность переноса краевых условий в конечный момент времени в любую точку интервала времени и формулируется теоремой 2 .

теорема 2. Если $\mathrm{C}(\mathrm{t})$ - оптимальное управление, изменяющее за данный промежуток времени $\tau_{1}>0$ кинетическую энергию системы на максимальную величину $\mathrm{T}\left(\tau_{1}\right)-\mathrm{T}_{(0)}=\mathrm{T}_{1 \max }, \quad$ то $\quad \mathrm{C}(\mathrm{t})=\stackrel{\circ}{\mathrm{C}}(\mathrm{t}) \quad$ есть оптимальное по быстродействию управление задачи демпфирования колебаний системы.

Доказательство осуществляется от противного по кинетической энергии. Пусть $\mathrm{C}(\mathrm{t})=\stackrel{\circ}{\mathrm{C}}-$ оптимальное управление, которое изменяет за время $\tau_{1}$ кинетическую энергию на максимальную величину, то есть $\mathrm{T}\left(\tau_{1}\right)-\mathrm{T}_{0}=\Delta \mathrm{T}_{1 \max } . \quad$ Тогда $\quad \mathrm{C}(\mathrm{t}) \rightarrow \stackrel{\circ}{\mathrm{C}}(\mathrm{t})$ есть оптимальное по быстродействию управление. Пусть это не так, то есть $C(t) \neq \stackrel{\circ}{C}$. Но тогда найдется управление $\widetilde{C}(t)$ такое, что изменение кинетической энергии на величину $\Delta \mathrm{T}_{1 \max }^{\prime}$ произойдет за время $\tau^{\prime}<\tau_{1}$. Противоречие доказывает сформулированное в теореме утверждение.

Далее, для перехода к управлению по текущему состоянию системы интервал кинетической энергии разбивают на ряд участков (14), причем в узловых точках значения

ISPC The Combination of Technology \&

Education, Östersund, Sweden кинетической энергии удовлетворяют строгим неравенствам. Этим узловым точкам соответствует временной ряд (15). Каждому участку соответствует и допустимое управление. Анализ ряда показывает, что начальные условия последующего интервала определяются конечными условиями предыдущего интервала (условия стыковки) (16), а краевые условия для сопряженных функций данного интервала условиями трансверсальности в правом конце (17).

При разбиении на бесконечное число участков сопряженные функции оказываются определенными по текущим значениям обобщенных координат и единственным множителем Лагранжа

$$
\begin{gathered}
{\left[\mathrm{T}_{(0)} \mathrm{T}_{(\mathrm{k})}\right] \rightarrow \mathrm{T}_{0}>\mathrm{T}_{1}>\ldots>\mathrm{T}_{\mathrm{n}} \ldots>\mathrm{T}_{\mathrm{N}}=\mathrm{T}_{(\mathrm{k})}} \\
{\left[0, t_{(k)}\right] \rightarrow t_{0}<t_{1}<\ldots<t_{n}<\ldots<t_{N}=t_{(k)}} \\
\psi_{\mathrm{i}}\left(\mathrm{t}_{\mathrm{n}}\right)=-\lambda \dot{\mathrm{z}}_{\mathrm{i}}\left(\mathrm{t}_{\mathrm{n}}\right), \\
z_{n+1}^{i}\left(t_{n}\right)=z_{n}^{i}\left(t_{n}\right) ; \dot{z}_{n+1}^{i}\left(t_{n}\right)=\dot{z}_{n}^{i}\left(t_{n}\right)
\end{gathered}
$$

\section{Если}

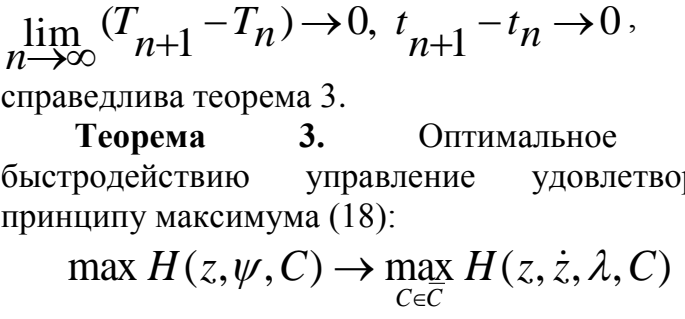

Полученный результат принципиально облегчает проблему расчета оптимальных алгоритмов управления жесткостью, т.к. в этом случае закон управления формируется по текущему значению обобщенных координат. С учетом доказанного, уравнение для определения оптимальных коэффициентов жесткости $\mathrm{c}_{\mathrm{ij}}$ амортизаторов представляется в виде

$$
c_{i j}=\left\{\begin{array}{l}
c_{i j \max }, \operatorname{signiz}^{i}\left(z^{i}-q_{i}\right)=+1 ; \\
c_{i j \min }, \operatorname{signz}^{i}\left(z^{i}-q_{i}\right)=-1 .
\end{array}\right.
$$

Способ демпфирования колебаний, основанный на управлении параметрами системы может оказаться трудно осуществимым или нецелесообразным, когда на систему будет воздействовать узкий спектр заранее известных нагрузок. В этом случае вытекает задача выбора таких постоянных параметров, при которых демпфирование осуществлялось бы наилучшим образом.

Целесообразно в этом случае в качестве критерия эффективности выбирать отклонение характеристик колебаний систем с постоянными 
параметрами от характеристик колебания систем c оптимально управляемыми параметрами (функционал точности) [9-10]. Постановка оптимизационной задачи в этом случае будет следующая: для колебательной системы описываемой дифференциальными уравнениями (20) с начальными условиями (21)определить такие параметры жесткости и сопротивления, чтобы функционал ошибки (22) принимал минимальное значение

$$
\begin{gathered}
\mathrm{A} \ddot{\mathrm{z}}=-[\mathrm{K} \dot{\mathrm{z}}+\mathrm{Cz}-\mathrm{q}(\mathrm{t}, \mathrm{C})]=\mathrm{F}(\mathrm{z}, \dot{\mathrm{z}}, \mathrm{C}, \mathrm{K}, \mathrm{t}) \\
\mathrm{t}=0, \quad \mathrm{z}=\mathrm{z}(0), \quad \dot{\mathrm{z}}=\dot{\mathrm{z}}_{(0)} \\
J=\frac{1}{2} \int_{0}^{t_{(k)}}(z-y)^{T} E(z-y) \cdot d t \rightarrow \min
\end{gathered}
$$

где $\mathrm{y}(\mathrm{t})$ - решение системы (20) при оптимальном управлении жесткостью, $\mathrm{K}=\left[\mathrm{k}_{\mathrm{ij}}\right]-$ матрица коэффициентов демпфирования, E - единичная матрица. Задание вектора $\mathrm{y}(\mathrm{t})$, как оптимального решения, характеризует предельно достижимое качество демпфирования.

Для решения задачи осуществляют расширение вектора обобщенных координат, подчинив параметры К и С дифференциальным связям:

$$
\dot{\mathrm{p}}=\mathrm{u},
$$

где $\mathrm{p} \in \mathrm{C} \cup \mathrm{K}$ - вектор параметров системы. u вектор управления, который удовлетворяет ограничениям:

$$
J_{1}=\frac{1}{2} \int_{0}^{t_{(k)}} u^{T} \text { Eudt, } U \rightarrow 0 ; t_{(k)} \rightarrow \infty
$$

Введем функции чувствительности фазовых координат к параметрам $\mathrm{p}=\left[\mathrm{p}_{\mathrm{S}}\right]$ $\Gamma_{s r}=\frac{\partial z_{s}}{\partial p_{r}}, s=\overline{1, N} ; r=\overline{1, N} ; N=n^{2}$

Для получения условий оптимальности вновь используется игольчатое варьирование управления u. При этом вариации управления u соответствует вариация параметра $\delta p$ и вариация обобщенной координаты

$$
\delta \mathrm{z}_{(\mathrm{p})}=\frac{\partial \mathrm{z}}{\partial \mathrm{p}} \delta \mathrm{p}=\Gamma \delta \mathrm{p}
$$

где $\Gamma=\left(\begin{array}{l}\Gamma_{11} \Gamma_{12} \ldots \Gamma_{1 \mathrm{~N}} \\ \ldots \ldots \ldots \ldots \ldots . . . \\ \Gamma_{\mathrm{n} 1} \Gamma_{\mathrm{n} 2} \ldots \Gamma_{\mathrm{nN}}\end{array}\right)-$ матрица функции чувствительности.

Функции чувствительности определяются по исходной системе дифференциальных уравнений:

$$
\begin{gathered}
\sum_{j=1}^{n}\left(A_{i j} \ddot{\Gamma}_{j r}+k_{i j} \dot{\Gamma}_{j r}+c_{i j} \Gamma_{j r}\right)+z^{r}=\frac{\partial q_{i}}{\partial p_{r}} \\
\mathrm{pr}_{\mathrm{r}} \in\left[\mathrm{c}_{\mathrm{ij}}\right], \quad \mathrm{i}, \mathrm{j}, \mathrm{s}, \mathrm{r}=\overline{1, \mathrm{n}} ; \quad \\
\left.\sum_{j=1}^{n}\left(A_{i j} \ddot{\Gamma}_{j r}+k_{i j} \dot{\Gamma}_{j r}+c_{i j} \Gamma_{j r}\right)+\dot{z}^{r}\right)=\frac{\partial q_{i}}{\partial p_{r}} \\
\mathrm{p}_{\mathrm{r}} \in\left[\mathrm{k}_{\mathrm{ij}}\right], \quad \mathrm{i}, \mathrm{j}, \mathrm{s}, \mathrm{r}=\overline{1, \mathrm{n}} ; \\
\mathrm{t}=0, \quad \Gamma_{\mathrm{jr}}=\dot{\Gamma}_{\mathrm{jr}}=0 .
\end{gathered}
$$

Введя теперь вектор сопряженных переменных для параметров р ,

$$
\lambda \equiv\left(\lambda_{1}, \lambda_{2} \ldots, \lambda_{\mathrm{N}}\right)^{\mathrm{T}}
$$

и множитель Лагранжа $\mu$, можно построить расширенный функционал

$$
\begin{aligned}
& \left.J^{*}=\frac{1}{2} \int_{0}^{t}(z) y\right)^{T} E(z-y) d t+ \\
& \left.+\mu \frac{1}{1} \int_{0}^{t} u^{T} \text { Eudt }+\int_{0}^{t} \lambda(p)-u\right) d t .
\end{aligned}
$$

Имея в виду, что обобщенные координаты являются неявными функциями параметров, после игольчатого варьирования получаются условия оптимальности управления u, параметров $\mathrm{p}$ и обобщенных координат $\mathrm{z}$, который формируется в виде теоремы.

Теорема 4. Для того, чтобы постоянные параметры $p \in C \bigcup K$ и соответствующие им обобщенные координаты z доставляли минимум функционалу точности (29), необходимо и достаточно существование непрерывной векторфункции $\lambda(t)$, удовлетворяющий сопряженной системе уравнений $\quad \dot{\lambda}+\mu(z-y)^{T} \Gamma=0 \quad$ и нулевым условиям трансверсальности $\mathrm{t}=\mathrm{t}_{(\mathrm{k})}, \quad \lambda=0, \quad$ и функция Гамильтона $H=-\lambda u+\frac{1}{2} \mu u^{T} E u=\max _{u \in \bar{R}} H(\lambda, u), \quad$ при любом $t \in\left\lfloor O, t_{(k)}\right\rfloor$ достигала своего максимального значения.

При этом функции чувствительности удовлетворяют дифференциальным уравнениям чувствительности (27).

Открытым здесь остается вопрос о практических расчетах по полученным соотношениям. Для получения удобных для расчета соотношений поступают так. Приращение параметра $\Delta \overline{\mathbf{p}}$ определяют 
двояким способом: как функцию приращения времени $\Delta t$, и как функцию приращения сопряженной переменной $\Delta \lambda$ путем его разложения в ряд по $\Delta t$ и $\Delta \lambda$ с удержанием величин второго порядка малости

$$
\Delta p=\frac{\partial p}{\partial t} \Delta t+\frac{\partial p}{\partial t} \Delta \lambda+\frac{\partial^{2} p}{\partial t \partial \lambda} \Delta t \Delta \lambda+\ldots
$$

и как приращение правой части дифференциального уравнения, причем $\Delta p \equiv 0$;

$$
\Delta \lambda=-\mu(z-y)^{T} \Gamma \Delta t-\mu \frac{\partial z}{\partial p} \Gamma \Delta \lambda \Delta t .
$$

После несложных математических преобразований получаются расчетные уравнения для параметров системы, решение которых сводится к постоянной величине,

$$
\begin{gathered}
\dot{p}+\mu S(z-y){ }^{T} \Gamma=0 \\
\dot{\mathrm{S}}+\mu \mathrm{S}^{\mathrm{T}} \Gamma \mathrm{S}=0, \\
t=0, p=0, S=S_{(0)}, t \rightarrow \infty, S \rightarrow 0, p \rightarrow \text { const } \\
\text { где } \quad \mathrm{S}=\frac{\partial \mathrm{p}}{\partial \lambda} \quad-\quad \text { матрица чувствительности }
\end{gathered}
$$
параметров к сопряженным переменным, причем $\mathrm{S}=\mathrm{S}^{\mathrm{T}}$ - симметричная матрица.

\section{References:}

1. Kuchvid RP (2001) Ispitaniya avtomobilya:uchebnik- Moscow: MGIU, 2001.$351 \mathrm{p}$.

2. Kovaleva AS (1990) Upravlenie kolebatelnymi i vibroudarnymi sistemami. Moscow-Nauka1990, -256 p.

3. Kostoglotov AI, Shevcova LA (1987) Optimalnoe vozbuzhdenie rezonansnykhkolebanij uprugikh system prikladnaya mekhanika, 1987, T.23 №6, pp.23-30.

4. Natshif A, Dzhouns D, Khenderson D (1988) Dempfirovanie kolebanij. Moscow-Mir-1988, $448 \mathrm{p}$.

5. Bishop R (1986) Kolebaniya /per s angl. pod red.-Ya.G Panovko.-3-e-izd -Moscow -Nauka1986, -190 p.

6. Komkov V (1975) Teoriya optimalnogo upravleniya dempfirovaniem kolebanij prostykh-uprugikh sistem, -Moscow -Mir-1975, $-158 \mathrm{p}$.

7. Vorobyev SA, Vorobyev SS, Reshenkin AS, Goncharov RA, Tihomirov AG (2015) The
Dynamic characteristics of vehicle suspension systems with optimal damping. ISJ Theoretical \& Applied Science 03 (23): 93-96. Soi: http://so-i.org/1.1/TAS*03(23)17 Doi: http://dx.doi.org/10.15863/TAS.2015.03.23.17

8. Vorobyev SA, Vorobyev SS, Reshenkin AS, Goncharov RA, Tihomirov AG (2015) Necessary conditions for optimal damping suspension car with dynamic action. ISJ Theoretical \& Applied Science 03 (23): 97-100. Soi: http://s-o-i.org/1.1/TAS*03(23)18 Doi: http://dx.doi.org/10.15863/TAS.2015.03.23.18

9. Vorobyev SA, Kostoglotov AI, Kulechov AV (2000) Mgnovenno-optimalnoe dempfirovanie kolebanyi mnogomassovych system c uprugimi svyazyami.-Izv.Vuzov, Sev-Kav. Region, Estesstvennye nauki, 2000, №2. pp. 29-32.

10. Vorobyev SA, Kostoglotov AI, Kulechov AV (2001) Sposob dempfirovaniya kolebanyi uprugopodvechennogo obyekta.. - Patent PF na izobreteniee №2162034 ot 20.01.01. 